\title{
Equivariant versions of higher order orbifold Euler characteristics *
}

\author{
S.M. Gusein-Zade ${ }^{\dagger} \quad$ I. Luengo $\quad$ A. Melle-Hernández ${ }^{\ddagger}$
}

\begin{abstract}
There are (at least) two different approaches to define an equivariant analogue of the Euler charateristic for a space with a finite group action. The first one defines it as an element of the Burnside ring of the group. The second approach emerged from physics and includes the orbifold Euler characteristic and its higher order versions. Here we give a way to merge the two approaches together defining (in a certain setting) higher order Euler characteristics with values in the Burnside ring of a group. We give Macdonald type equations for these invariants. We also offer generalized ("motivic") versions of these invariants and formulate Macdonald type equations for them as well.
\end{abstract}

\section{Introduction}

Let $X$ be a topological space (good enough, say, a real subanalytic variety) with an action of a finite group $G$. There are (at least) two different approaches to define an equivariant analogue of the Euler charateristic for the

*Math. Subject Class.: 55M35, 32Q55, 19A22. Keywords: finite group actions, orbifold Euler characteristic, Burnside ring, complex quasi-projective varieties, wreath products, generating series.

${ }^{\dagger}$ The work of the first author (Sections 1, 2 and 4) was supported by the grant 16-1110018 of the Russian Science Foundation. Address: Moscow State University, Faculty of Mathematics and Mechanics, GSP-1, Moscow, 119991, Russia. E-mail: sabir@mccme.ru

¥The last two authors were partially supported by the grant MTM2013-45710-C02-02-P. Address: ICMAT (CSIC-UAM-UC3M-UCM); Complutense University of Madrid, Dept. of Algebra, Madrid, 28040, Spain. E-mail: iluengo@mat.ucm.es, amelle@mat.ucm.es 
pair $(X, G)$. The first one $([27])$ defines the equivariant Euler characteristic $\chi^{G}(X)$ as an element of the Burnside ring $A(G)$ of the group $G$. The second approach emerged from physics (the string theory of orbifolds: [10], [11]). The orbifold Euler characteristic $\chi^{\text {orb }}(X, G)$ is defined through the fixed point sets of some subgroups of $G$ and is an integer. Higher order (orbifold) Euler characteristics were introduced in [1] and [8] (also as integers). They can be defined through the fixed point sets of collections of commuting elements in $G$. Here we give a way to merge the two approaches together.

Through this paper we consider the Euler characteristic $\chi(\cdot)$ defined as the alternating sum of the dimensions of the cohomology groups with compact support. This Euler characteristic is not a homotopy invariant in the usual sense, but an invariant of the homotopy type defined in terms of proper maps. It is an additive function on the algebra of ("good") spaces. For complex quasi-projective varieties this Euler characteristic coincides with the "usual" one, i. e., with the alternating sum of the dimensions of the usual cohomology groups.

There is the universal additive invariant on the algebra of complex constructible sets. It takes values in the Grothendieck ring $K_{0}\left(\operatorname{Var}_{\mathbb{C}}\right)$ of complex quasi-projective varieties and can be regarded as a generalized ("motivic") Euler characteristic $\chi_{g}(X)$. There were defined generalized ("motivic") analogues of the orbifold Euler characteristic and of its higher order generalizations. First it was essentially made in [2] (for the Hodge-Deligne polynomial) and then formulated precisely in [17] and [19]. These higher order generalized Euler characteristics take values in a certain modification of the Grothendieck ring $K_{0}\left(\operatorname{Var}_{\mathbb{C}}\right)$ of complex quasi-projective varieties.

The Euler characteristic satisfies the Macdonald type equation

$$
1+\sum_{n=1}^{\infty} \chi\left(S^{n} X\right) \cdot t^{n}=(1-t)^{-\chi(X)}
$$

where $S^{n} X=X^{n} / S_{n}$ is the $n$th symmetric power of $X$ (see, e.g., [23]). A Macdonald type equation for a given invariant expresses the generating series of the values of the invariant for the symmetric powers of a space (or for their analogues) as a series not depending on the space (in this case $(1-t)^{-1}$ ) with the exponent equal to the value of the invariant for the space itself. If the invariant takes values in a ring $R$ different from a number ring (i.e., from $\mathbb{Z}$, $\mathbb{Q}, \mathbb{R}$ or $\mathbb{C}$ ), Macdonald type equations can be formulated in terms of a socalled power structure over the ring. There are Macdonald type equations for 
the orbifold Euler characteristic and for its higher order analogues ([29], [28]) and also for the equivariant Euler characteristic with values in the Burnside ring $A(G)$ of $G$ (see Lemma 1 below). An analogue of these equations for the generalized ("motivic") higher order Euler characteristics was obtained in [19]. It was formulated in terms of the (natural) power structure over the Grothendieck ring $K_{0}\left(\operatorname{Var}_{\mathbb{C}}\right)$ of complex quasi-projective varieties: 15. (Its version for the orbifold Hodge-Deligne polynomial was proved in [30.)

Here we define higher order Euler characteristics with values in the Burnside ring of a group. This is made in the setting when there are two commuting finite group actions. Then one of them can be treated in the way similar to "the orbifold aproach" and the other in the way which leads to invariants (say, equivariant Euler characteristic) with values in the Burnside ring of the group. This situation can be met, for example, in the following considerations. Assume that $(f, G)$ is a pair consisting of a quasi-homogeneous polynomial $f$ and an abelian group $G$ of its (diagonal) symmetries. Such pairs are subjects of analysis, in particular, in the Berglund-Hübsch-Henningson mirror symmetry [3], [4]. The classical monodromy transformation of $f$ is a map of a finite order from the Milnor fibre of $f$ into itself. It commutes with the $G$-action. Thus one has the action of two groups: the group $G$ and the (cyclic) group generated by the monodromy transformation. A study of the orbifold monodromy zeta function of $f$ leads to the situation when one should apply "the orbifold approach" to the first action whence the other one should be treated in another way, see, e. g., [12. We give Macdonald type equations for the constructed invariants. We also offer generalized ("motivic") versions of these invariants and formulate Macdonald type equations for them as well.

\section{Power structures over rings}

As it was indicted above, a Macdonald type equation for an invariant can be formulated in terms of a power structure over the ring of values of the invariant. Let $R$ be a commutative associative ring with unity. A power structure over the ring $R$ gives sense to expressions of the form $(A(t))^{m}$, where $A(t)=1+a_{1} t+a_{2} t^{2}+\ldots$ is a power series with the coefficients $a_{i}$ from $R$ and $m$ is an element of $R$.

Definition: A power structure over the $\operatorname{ring} R$ is a map $(1+t R[[t]]) \times R \rightarrow$ $1+t R[[t]]\left((A(t), m) \mapsto(A(t))^{m}\right)$ possessing the following properties:

1) $\left(1+a_{1} t+\ldots\right)^{m}=1+m a_{1} t+\ldots$; 
2) $(A(t) B(t))^{m}=(A(t))^{m}(B(t))^{m}$;

3) $(A(t))^{m+n}=(A(t))^{m}(A(t))^{n}$;

4) $(A(t))^{m n}=\left((A(t))^{m}\right)^{n}$.

Let $\mathfrak{m}$ be the ideal $t R[[t]]$ in the ring $R[[t]]$.

Definition: A power structure over the ring $R$ is finitely determined if the fact that $A(t) \in 1+\mathfrak{m}^{k}$ implies that $(A(t))^{m} \in 1+\mathfrak{m}^{k}$.

The natural power structure over the ring $\mathbb{R}$ of integers is defined by the standard formula for a power of a series (see, e. g., [26]):

$$
\begin{aligned}
& \left(1+a_{1} t+a_{2} t^{2}+\ldots\right)^{m}= \\
= & 1+\sum_{k=1}^{\infty}\left(\sum_{\left\{k_{i}\right\}: \sum i k_{i}=k} \frac{m(m-1) \cdots\left(m-\sum_{i} k_{i}+1\right) \cdot \prod_{i} a_{i}^{k_{i}}}{\prod_{i} k_{i} !}\right) \cdot t^{k} .
\end{aligned}
$$

An important example of a power structure over the Grothendieck ring of quasi-projective varieties was introduced in [15].

A quasi-projective variety is the complement of a projective variety in a projective one. The Grothendieck ring $K_{0}\left(\operatorname{Var}_{\mathbb{C}}\right)$ of complex quasi-projective varieties is the abelian group generated by the classes of complex quasiprojective varieties modulo the relations:

1) if $X$ and $X^{\prime}$ are isomorphic, then $[X]=\left[X^{\prime}\right]$;

2) if $Y \subset X$ is a Zariski closed subset, then $[X]=[Y]+[X \backslash Y]$.

The multiplication in $K_{0}\left(\operatorname{Var}_{\mathbb{C}}\right)$ is defined by the cartesian product. The class $\mathbb{L}=\left[\mathbb{A}_{\mathbb{C}}^{1}\right]$ of the complex affine line plays a special role in a number of constructions connected with the Grothendieck ring $K_{0}\left(\operatorname{Var}_{\mathbb{C}}\right)$. (In [6] it was shown that $\mathbb{L}$ is a divisor of zero in $K_{0}\left(\operatorname{Var}_{\mathbb{C}}\right)$.)

A power structure over the ring $K_{0}\left(\operatorname{Var}_{\mathbb{C}}\right)$ is defined in [15] by the formula

$$
\begin{aligned}
& \left(1+\left[A_{1}\right] t+\left[A_{2}\right] t^{2}+\ldots\right)^{[M]}= \\
= & 1+\sum_{k=1}^{\infty}\left(\sum_{\left\{k_{i}\right\}: \sum i k_{i}=k}\left[\left(\left(M^{\sum_{i} k_{i}} \backslash \Delta\right) \times \prod_{i} A_{i}^{k_{i}}\right) / \prod_{i} S_{k_{i}}\right]\right) \cdot t^{k},(2)
\end{aligned}
$$

where $A_{i}, i=1,2, \ldots$, and $M$ are quasi-projective varieties ([ $\left.A_{i}\right]$ and $[M]$ are their classes in the ring $\left.K_{0}\left(\operatorname{Var}_{\mathbb{C}}\right)\right), \Delta$ is the large diagonal in $M^{\sum_{i} k_{i}}$, 
i. e., the set of (ordered) $\left(\sum_{i} k_{i}\right)$-tuples of points of $M$ with at least two coinciding ones, the group $S_{k_{i}}$ of permutations on $k_{i}$ elements acts by the simultaneous permutations on the components of the corresponding factor $M^{k_{i}}$ in $M^{\sum_{i} k_{i}}=\prod_{i} M^{k_{i}}$ and on the components of $A_{i}^{k_{i}}$.

One can see that the coefficient at $t^{k}$ in the right hand side of (2) has the following interpretation. Let $I: \coprod_{i=1}^{\infty} A_{i} \rightarrow \mathbb{Z}$ be the "tautological function" on the disjoint union $\coprod_{i=1}^{\infty} A_{i}$ sending $A_{i}$ to $i$. The coefficient at $t^{k}$ in the right hand side of (2) is represented by the configuration space of pairs $(K, \psi)$, where $K$ is a finite subset of $M$ and $\psi$ is a map $K \rightarrow \coprod_{i=1}^{\infty} A_{i}$ such that $\sum_{x \in K} I(\psi(x))=$ $k$. This interpretation makes it much easier to prove that the equation (2) really defines a power structure (i. e., to verify the properties 2)-4) from the definition) and also permits to prove some formulae for generating series of classes of Hilbert schemes of "fat points" (zero-dimensional subschemes) on quasi-projective manifolds (see, e. g., [16]). Less formally, see [14], [9], one can say that on the variety $M$ there live particles equipped with some natural numbers (multiplicities, masses, charges, ...). A particle of multiplicity $n$ has a complicated space of internal states which is parametrized by points of a quasi-projective variety $A_{n}$ and the coefficient at $t^{k}$ is the configuration space of tuples of particles with the total multiplicity $k$.

The notion of a power structure over a ring is closely related with the notion of a $\lambda$-ring structure. A $\lambda$-ring structure (or a pre- $\lambda$-ring structure in a certain terminology) is an additive-to-multiplicative homomorphism $R \rightarrow$ $1+t R[[t]], a \mapsto \lambda_{a}(t)$ such that $\lambda_{a}(t)=1+a t+\ldots$, where the term additiveto-multiplicative means that $\lambda_{a+b}(t)=\lambda_{a}(t) \cdot \lambda_{b}(t)$. A $\lambda$-ring structure over a ring defines a finitely determined power structure over it in the following way. Any series $A(t) \in 1+t R[[t]]$ can be in a unique way represented as $\prod_{i=1}^{\infty} \lambda_{b_{i}}\left(t^{i}\right)$, for some $b_{i} \in R$. Then one defines $(A(t))^{m}:=\prod_{i=1}^{\infty} \lambda_{m b_{i}}\left(t^{i}\right)$. On the other hand, in general, there are many $\lambda$-ring structures corresponding to one power structure over a ring. One can show that the power strucure (2) is defined by the $\lambda$-ring structure on the Grothendieck ring $K_{0}\left(\operatorname{Var}_{\mathbb{C}}\right)$ given by the Kapranov zeta function

$$
\zeta_{[M]}(t):=1+\sum_{n=1}^{\infty}\left[S^{n} M\right] \cdot t^{n},
$$

where $S^{n} M$ is the n-th symmetric power of the variety $M$. This follows for 
the following equation

$$
\zeta_{[M]}=(1-t)^{-[M]}=\left(1+t+t^{2}+\ldots\right)^{[M]} .
$$

Burnside ring $A(G)$ of a finite group $G$ is the Grothendieck ring of finite $G$-sets: see, e.g., [7]). As an abelian group the Burnside ring $A(G)$ is freely generated by the classes $[G / H]$ of the quotients $G / H$ for representatives $H$ of the conjugacy classes $\mathfrak{h}$ of subgroups of $G$. The multiplication is defined by the cartesian product with the diagonal action of $G$. There is a natural power structure over the Burnside ring $A(G)$ : see, e.g., [18]. This power structure is defined in a way similar to that one over the ring $K_{0}\left(\operatorname{Var}_{\mathbb{C}}\right)$. Namely, if $A_{i}, i=1,2, \ldots$, and $M$ are finite $G$-sets, the same equation (2) defines the series $\left(1+\left[A_{1}\right] t+\left[A_{2}\right] t^{2}+\ldots\right)^{[M]}$ where the action of the group $G$ on the summands is the natural (the diagonal) one. In particular, for a finite $G$-set $X$ one has

$$
(1-t)^{-[X]}=1+[X] t+\left[S^{2} X\right] t^{2}+\left[S^{3} X\right] t^{3}+\ldots,
$$

where $S^{k} X=X^{k} / S_{k}$ is the $k$ th symmetric power of the $G$-set $X$ with the diagonal action of $G$.

Let $X$ be a $G$-space. For a point $x \in X$, let $G_{x}=\{g \in G: g \cdot x=x\}$ be the isotropy subgroup of the point $x$. For a subgroup $H \subset G$, let $X^{H}=$ $\{x \in X: H x=x\}$ be the fixed point set of $H\left(X^{H}=\left\{x \in X: H \subset G_{x}\right\}\right)$ and let $X^{(H)}=\left\{x \in X: G_{x}=H\right\}$ be the set of points with the isotropy group $H$. Let Conjsub $G$ be the set of the conjugacy classes of subgroups of $G$. For a conjugacy class $\mathfrak{h} \in \operatorname{Conjsub} G$, let $X^{\mathfrak{h}}=\{x \in X: x \in$ $X^{H}$ for a subgroup $\left.H \in \mathfrak{h}\right\}, X^{(\mathfrak{h})}=\left\{x \in X: G_{x} \in \mathfrak{h}\right\}$.

The equivariant Euler characteristic of a (good enough) $G$-space $X$ is defined by

$$
\chi^{G}(X):=\sum_{\mathfrak{h} \in \operatorname{Conjsub} G} \chi\left(X^{(\mathfrak{h})} / G\right)[G / H] \in A(G),
$$

where $H$ is a representative of the conjugacy class $\mathfrak{h}$ (see, e. g., [27]). 


\section{Higher order Euler characteristics \& Mac- donald type equations}

The orbifold Euler characteristic $\chi^{\text {orb }}(X, G)$ of the $G$-space $X$ is defined, e.g., in [1], [21]:

$$
\chi^{\text {orb }}(X, G)=\frac{1}{|G|} \sum_{\substack{\left.g_{0}, g_{1}\right) \in G \times G: \\ g_{0} g_{1}=g_{1} g_{0}}} \chi\left(X^{\left\langle g_{0}, g_{1}\right\rangle}\right)=\sum_{[g] \in G_{*}} \chi\left(X^{\langle g\rangle} / C_{G}(g)\right) \in \mathbb{Z},
$$

where $G_{*}$ is the set of the conjugacy classes of elements of $G, g$ is a representative of the class $[g], C_{G}(g)=\left\{h \in G: h^{-1} g h=g\right\}$ is the centralizer of $g$, and $\langle g\rangle$ and $\left\langle g_{0}, g_{1}\right\rangle$ are the subgroups of $G$ generated by the corresponding elements.

The higher order Euler characteristics of $(X, G)$ are defined by:

$$
\chi^{(k)}(X, G)=\frac{1}{|G|} \sum_{\substack{\mathbf{g} \in G^{k+1} \\ g_{i} g_{j}=g_{j} g_{i}}} \chi\left(X^{\langle\mathbf{g}\rangle}\right)=\sum_{[g] \in G_{*}} \chi^{(k-1)}\left(X^{\langle g\rangle}, C_{G}(g)\right),
$$

where $\mathbf{g}=\left(g_{0}, g_{1}, \ldots, g_{k}\right),\langle\mathbf{g}\rangle$ is the subgroup generated by $g_{0}, g_{1}, \ldots, g_{k}$, and (for the second, recurrent, definition) $\chi^{(0)}(X, G)$ is defined as the usual Euler characteristic $\chi(X / G)$ of the quotient. The orbifold Euler characteristic $\chi^{\text {orb }}(X, G)$ is the Euler characteristic $\chi^{(1)}(X, G)$ of order 1 .

For a $G$-space $X$, the cartesian power $X^{n}$ carries the natural action of the wreath product $G_{n}=G \curlywedge S_{n}=G^{n} \rtimes S_{n}$ generated by the natural action of the symmetric group $S_{n}$ (permutting the factors) and by the natural (componentwise) action of the cartesian power $G^{n}$. The pair $\left(X_{n}, G_{n}\right)$ should be (or can be) considered as an analogue of the symmetric power of the pair $(X, G)$. One has the following Macdonald type equation (see [28, Theorem $\mathrm{A}])$

$$
\sum_{n \geq 0} \chi^{(k)}\left(X^{n}, G_{n}\right) \cdot t^{n}=\left(\prod_{r_{1}, \ldots, r_{k} \geq 1}\left(1-t^{r_{1} r_{2} \cdots r_{k}}\right)^{r_{2} r_{3}^{2} \cdots r_{k}^{k-1}}\right)^{-\chi^{(k)}(X, G)}
$$

When $k=0$, one gets the equation (11) for the quotient $X / G$.

The equation (6) can be interpreted in the following way. Set

$$
\mathbb{X}_{(X, G)}^{(k)}(t):=\sum_{n \geq 0} \chi^{(k)}\left(X^{n}, G_{n}\right) \cdot t^{n}
$$


For the natural action of the group $G$ on $G$ (regarded as a zero-dimensional finite space) one has $\chi^{(k)}(G, G)=1$. Therefore

$$
\mathbb{X}_{(G, G)}^{(k)}(t)=\left(\prod_{r_{1}, \ldots, r_{k} \geq 1}\left(1-t^{r_{1} r_{2} \cdots r_{k}}\right)^{r_{2} r_{3}^{2} \cdots r_{k}^{k-1}}\right)^{-1}
$$

and thus

$$
\mathbb{X}_{(X, G)}^{(k)}(t)=\left(\mathbb{X}_{(G, G)}^{(k)}(t)\right)^{\chi^{(k)}(X, G)}
$$

Let $K_{0}\left(\operatorname{Var}_{\mathbb{C}}\right)\left[\mathbb{L}^{s}\right]_{s \in \mathbb{Q}}$ be the modification of the Grothendieck ring $K_{0}\left(\operatorname{Var}_{\mathbb{C}}\right)$ obtained by adding all rational powers of $\mathbb{L}$. (This includes $\mathbb{L}^{-1}$ and thus $K_{0}\left(\operatorname{Var}_{\mathbb{C}}\right)\left[\mathbb{L}^{s}\right]_{s \in \mathbb{Q}}$ contains the localization $K_{0}\left(\operatorname{Var}_{\mathbb{C}}\right)_{(\mathbb{L})}$ of the ring $K_{0}\left(\operatorname{Var}_{\mathbb{C}}\right)$ by $\mathbb{L}$. Pay attention that $\mathbb{L}$ is a zero divisor in $K_{0}\left(\operatorname{Var}_{\mathbb{C}}\right)([6])$ and therefore the natural map $K_{0}\left(\operatorname{Var}_{\mathbb{C}}\right) \rightarrow K_{0}\left(\operatorname{Var}_{\mathbb{C}}\right)_{(\mathbb{L})}$ is not injective. Therefore the natural map $K_{0}\left(\operatorname{Var}_{\mathbb{C}}\right) \rightarrow K_{0}\left(\operatorname{Var}_{\mathbb{C}}\right)\left[\mathbb{L}^{s}\right]_{s \in \mathbb{Q}}$ is not injective as well.) A power structure on the ring $K_{0}\left(\operatorname{Var}_{\mathbb{C}}\right)\left[\mathbb{L}^{s}\right]_{s \in \mathbb{Q}}$ can be defined as a sort of an extension of that on $K_{0}\left(\operatorname{Var}_{\mathbb{C}}\right)$ using the following equations:

1) $\left(A\left(\mathbb{L}^{s} t\right)\right)^{[M]}=\left.(A(t))^{[M]}\right|_{t \mapsto \mathbb{L}^{s} t}$;

2) $\zeta_{\mathbb{L}^{s}[M]}(t)=\zeta_{[M]}\left(\mathbb{L}^{s} t\right)$.

The second equation permits to define $(A(t))^{\mathbb{L}^{s}[M]}$. One has to represent $A(t)$ as $\prod_{i=1}^{\infty} \zeta_{b_{i}}\left(t^{i}\right), b_{i} \in K_{0}\left(\operatorname{Var}_{\mathbb{C}}\right)$, and then to define $(A(t))^{\mathbb{L}^{s}[M]}$ by

$$
(A(t))^{\mathbb{L}^{s}[M]}:=\prod_{i=1}^{\infty} \zeta_{b_{i}[M]}\left(\mathbb{L}^{s} t^{i}\right) .
$$

Remark. If, in the considerations bellow, one uses only non-negative weights $\varphi_{i}$, one can work with the ring $K_{0}\left(\operatorname{Var}_{\mathbb{C}}\right)\left[\mathbb{L}^{s}\right]_{s \in \mathbb{Q}>0}$. The natural map $K_{0}\left(\operatorname{Var}_{\mathbb{C}}\right) \rightarrow$ $K_{0}\left(\operatorname{Var}_{\mathbb{C}}\right)\left[\mathbb{L}^{s}\right]_{s \in \mathbb{Q} \geq 0}$ is injective.

Now let $X$ be a smooth quasi-projective variety of dimension $d$ with an (algebraic) action of the group $G$. To define the higher order generalized (orbifold) Euler characteristics of the pair $(X, G)$, one has to use the so called age (or fermion shift) $F_{x}^{g}$ of an element $g \in G$ at a fixed point $x$ of $g$ defined in [31], [20]. The element $g$ acts on the tangent space $T_{x} X$ as an automorphism of finite order. This action on $T_{x} X$ can be represented by a diagonal matrix $\operatorname{diag}\left(\exp \left(2 \pi i \theta_{1}\right), \ldots, \exp \left(2 \pi i \theta_{d}\right)\right)$ with $0 \leq \theta_{j}<1$ for 
$j=1,2, \ldots, d\left(\theta_{j}\right.$ are rational numbers). The age of the element $g$ at the point $x$ is defined by $F_{x}^{g}=\sum_{j=1}^{d} \theta_{j} \in \mathbb{Q}_{\geq 0}$.

For $g \in G$, let the number of $C_{G}(g)$-orbits in the the set of connected components of the fixed point set $X^{\langle g\rangle}$ be equal to $N_{g}$, and let $X_{1}^{\langle g\rangle}, X_{2}^{\langle g\rangle}, \ldots$, $X_{N_{g}}^{\langle g\rangle}$ be the unions of the components of each of the orbits. For $1 \leq \alpha_{g} \leq N_{g}$, let $F_{\alpha_{g}}^{g}$ be the age $F_{x}^{g}$ at a point of $X_{\alpha_{g}}^{\langle g\rangle}$ (this age does not depend on the point $x \in X_{\alpha_{g}}^{\langle g\rangle}$ ).

For a rational number $\varphi_{1} \in \mathbb{Q}$, the generalized orbifold Euler characteristic of weight $\varphi_{1}$ of the pair $(X, G)$ is defined by

$$
[X, G]_{\varphi_{1}}:=\sum_{[g] \in G_{*}} \sum_{\alpha_{g}=1}^{N_{g}}\left[X_{\alpha_{g}}^{\langle g\rangle} / C_{G}(g)\right] \cdot \mathbb{L}^{\varphi_{1} F_{\alpha_{g}}^{\langle g\rangle}} \in K_{0}\left(\operatorname{Var}_{\mathbb{C}}\right)\left[\mathbb{L}^{s}\right]_{s \in \mathbb{Q}} .
$$

For $\varphi_{1}=1$ one gets the definition of the generalized orbifold Euler characteristic from [17] inspired by the definition of the orbifold Hodge-Deligne polynomial from [2]. (This generalized orbifold Euler characteristic maps to the orbifold Hodge-Deligne polynomial by the natural ring homomorphism $\left.e: K_{0}\left(\operatorname{Var}_{\mathbb{C}}\right) \rightarrow \mathbb{Z}[u, v].\right)$ For $\varphi_{1}=0$ one gets the so called inertia stack class: see, e.g., [13.

For a subgroup $H \subset G$, and for an $H$-invariant submanifold $Y \subset X$, let us define $[Y, H]_{X, \varphi_{1}}$ by

$$
[Y, H]_{X, \varphi_{1}}:=\sum_{[g] \in H_{*}} \sum_{\alpha_{g}=1}^{N_{g}}\left[Y_{\alpha_{g}}^{\langle g\rangle} / C_{H}(g)\right] \cdot \mathbb{L}^{\varphi_{1} F_{\alpha g}^{\langle g\rangle}} \in K_{0}\left(\operatorname{Var}_{\mathbb{C}}\right)\left[\mathbb{L}^{s}\right]_{s \in \mathbb{Q}},
$$

where $Y_{\alpha_{g}}^{\langle g\rangle}=X_{\alpha_{g}}^{\langle g\rangle} \cap Y, F_{\alpha_{g}}^{\langle g\rangle}$ is the age of $g$ at a point of $X_{\alpha_{g}}^{\langle g\rangle}$. (Pay attention that the age of an element $g$ is determined from its action on $T_{x} X$.)

Let $\varphi=\left(\varphi_{1}, \varphi_{2}, \ldots\right)$ be a fixed sequence of rational numbers. The generalized (orbifold) Euler characteristics of order $k$ of weight $\underline{\varphi}$ of the pair $(X, G)$ is defined recursively by

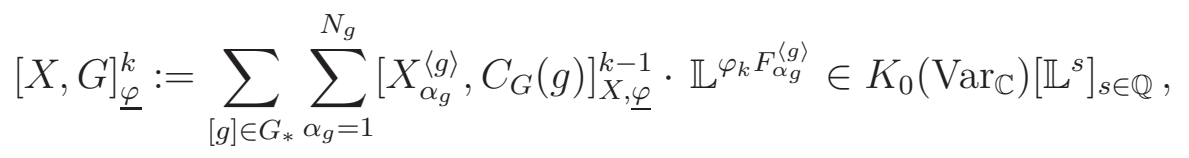

where, for an $H$-invariant submanifold $Y \subset X(H \subset G),[Y, H]_{X, \underline{\varphi}}^{1}:=$ $[Y, H]_{X, \varphi_{1}}$ is defined by (8). (Alternatively one can start from $k=\overline{0}$ using the definition $[Y, H]_{X, \underline{\varphi}}^{0}=[Y, H]^{0}=[Y / H]$.) 
One has the following Macdonald type equations ([19])

$$
\sum_{n \geq 0}\left[X^{n}, G_{n}\right]_{\underline{\varphi}}^{k} \cdot t^{n}=\left(\prod_{r_{1}, \ldots, r_{k} \geq 1}\left(1-\mathbb{L}^{\Phi_{k}(\underline{r}) d / 2} \cdot t^{r_{1} r_{2} \cdots r_{k}}\right)^{r_{2} r_{3}^{2} \cdots r_{k}^{k-1}}\right)^{-[X, G]_{\underline{\varphi}}^{k}},
$$

where

$$
\Phi_{k}\left(r_{1}, \ldots, r_{k}\right)=\varphi_{1}\left(r_{1}-1\right)+\varphi_{2} r_{1}\left(r_{2}-1\right)+\ldots+\varphi_{k} r_{1} r_{2} \cdots r_{k-1}\left(r_{k}-1\right) .
$$

\section{Equivariant higher order Euler character- istics}

Assume that $X$ is a (good enough) topological space with commuting actions of two finite groups $G_{O}$ and $G_{B}$ (or equivalently with an action of the product $\left.G_{O} \times G_{B}\right)$. The quotient $X / G_{O}$ carries the natural $G_{B}$-action and thus one can define $\chi^{\left(0, G_{B}\right)}\left(X ; G_{O}, G_{B}\right)$ as $\chi^{G_{B}}\left(X / G_{O}\right) \in A\left(G_{B}\right)$.

For an element $g \in G_{O}$, the fixed point set $X^{\langle g\rangle}$ is $G_{B}$-invariant and the quotient $X^{\langle g\rangle} / C_{G_{O}}(g)$ by the centralizer $C_{G_{O}}(g)$ carries the natural $G_{B^{-}}$ action.

Definition: The equivariant orbifold Euler characteristics of $\left(X ; G_{O}, G_{B}\right)$ is

$$
\begin{aligned}
\chi^{\left(1, G_{B}\right)}\left(X ; G_{O}, G_{B}\right) & :=\sum_{[g] \in G_{O *}} \chi^{G_{B}}\left(X^{\langle g\rangle} / C_{G_{O}}(g)\right) \\
& =\sum_{[g] \in G_{O *}} \chi^{\left(0, G_{B}\right)}\left(X^{\langle g\rangle} ; C_{G_{O}}(g), G_{B}\right) \in A\left(G_{B}\right) .
\end{aligned}
$$

The equivariant higher order Euler characteristics are defined recursively in the same way as the non-equivariant one.

Definition: The equivariant Euler characteristics of order $k$ of $\left(X ; G_{O}, G_{B}\right)$ is

$$
\chi^{\left(k, G_{B}\right)}\left(X ; G_{O}, G_{B}\right):=\sum_{[g] \in G_{O *}} \chi^{\left(k-1, G_{B}\right)}\left(X^{\langle g\rangle} ; C_{G_{O}}(g), G_{B}\right) \in A\left(G_{B}\right) .
$$


Definition (12) is convinient for the proof of Theorem 1, Iterating (12) one gets the following equation for the equivariant higher order Euler characteristics:

$$
\chi^{\left(k, G_{B}\right)}\left(X ; G_{O}, G_{B}\right)=\sum_{[\phi] \in \operatorname{Hom}\left(\mathbb{Z}^{k}, G_{O}\right) / G_{O}} \chi^{G_{B}}\left(X^{\langle\phi\rangle} / C_{G_{O}}(\phi)\right),
$$

where the group $G_{O}$ acts on $\operatorname{Hom}\left(\mathbb{Z}^{k}, G_{O}\right)$ by conjugation, $X^{\langle\phi\rangle}$ is the fixed point set of the image of $\phi, C_{G_{O}}(\phi)=\left\{g \in G_{O}: g^{-1} \phi g=\phi\right\}$.

Theorem 1 One has

$\sum_{n \geq 0} \chi^{\left(k, G_{B}\right)}\left(X^{n} ;\left(G_{O}\right)_{n}, G_{B}\right) \cdot t^{n}=\left(\prod_{r_{1}, \ldots, r_{k} \geq 1}\left(1-t^{r_{1} r_{2} \cdots r_{k}}\right)^{r_{2} r_{3}^{2} \cdots r_{k}^{k-1}}\right)^{-\chi^{\left(k, G_{B}\right)}\left(X ; G_{O}, G_{B}\right)}$

where the exponent in the right hand side is defined by the power structure over the Burside ring $A\left(G_{B}\right)$.

Proof. The proof essentially repeats, e.g., the one in [28] (see also[19]). One has to pay attention to two facts. First, all subsets participating in the course of the proof in [28] are $G$-invariant. Second, one has to use the Macdonald type equation for the equivariant Euler characteristic $\chi^{G}(\cdot)$ : see (15) below.

The Macdonald type equation for the equivariant Euler characteristic must be known. However, we have not found it in the literature. Therefore we put its proof here.

Lemma 1 For a G-space $X$ one has

$$
1+\sum_{n=1}^{\infty} \chi^{G}\left(S^{n} X\right) \cdot t^{n}=(1-t)^{-\chi^{G}(X)} \in 1+t A(G)[[t]]
$$

where the right hand side is defined by the power structure over the Burnside ring $A(G)$ (or by the $\lambda$-structure on it).

Remark. Note that, for a finite $G$-set $X$, the equation (15) is just the definition of its right hand side. (In this case $\chi^{G}(X)=[X] \in A(G)$.) 
Proof. Let us denote the left hand side of (15) by $\chi^{G} \zeta_{X, G}(t)$. If $X=$ $X_{1} \coprod X_{2}$ is a decomposition of $X$ into two $G$-subspaces, one has

$$
\chi^{G} \zeta_{X, G}(t)=\chi^{G} \zeta_{X_{1}, G}(t) \cdot \chi^{G} \zeta_{X_{2}, G}(t)
$$

(This follows from the identities $S^{n} X=\coprod_{m=0}^{n}\left(S^{m} X_{1}\right) \times\left(S^{n-m} X_{2}\right), \chi^{G}(X \times$ $Y)=\chi^{G}(X) \chi^{G}(Y)$.) Therefore it is sufficient to prove (15) for the elements of a decomposition of $X$ into $G$-invariant subspaces. A "good enough" $G$ space (say, a real subanalytic one) can be represented as the disjoint union of subspaces of the form $\sigma^{d} \times(G / H)$, where $H$ is a subgroup of $G, G / H$ is the corresponding $G$-set (the quotient), and $\sigma^{d}$ is the open cell of dimension $d$ with the trivial $G$-action. Let $\bar{\sigma}^{d} \supset \sigma^{d}$ be the closed $d$-dimensional ball. Since $\bar{\sigma}^{d}$ can be $G$-equivariantly contructed to a point, $S^{k}\left(\bar{\sigma}^{d} \times(G / H)\right)$ can be contracted to $S^{k}(G / H)$. Therefore

$$
\chi^{G}\left(S^{k}\left(\bar{\sigma}^{d} \times(G / H)\right)\right)=\chi^{G}\left(S^{k}(G / H)\right)=\left[S^{k}(G / H)\right]
$$

(see, e.g., [27] where this is formulated for finite $G$ - $C W$-complexes) and thus $\chi^{G} \zeta_{S^{k}\left(\bar{\sigma}^{d} \times(G / H)\right)}(t)=1+\sum_{i=1}^{\infty}\left[S^{i}(G / H)\right] t^{i}=(1-t)^{-[G / H]}=(1-t)^{-\chi^{G}\left(\bar{\sigma}^{d} \times(G / H)\right)}$.

The equation (15) obviously holds for $X=\sigma^{d} \times(G / H)$ with $d=0$ (when $\sigma^{d}$ is a point). Assume that it holds for $X=\sigma^{d} \times(G / H)$ with $d<d_{0}$. One has a decomposition $\sigma^{d_{0}}=\sigma^{d_{0}} \coprod \sigma^{d_{0}} \coprod \sigma^{d_{0}-1}$. Therefore

$$
\begin{gathered}
\chi^{G} \zeta_{\sigma^{d_{0} \times(G / H)}}(t)=\left(\chi^{G} \zeta_{\sigma^{d_{0} \times(G / H)}}(t)\right)^{2} \cdot \chi^{G} \zeta_{\sigma^{\left(d_{0}-1\right)} \times(G / H)}(t) \\
\chi^{G} \zeta_{\sigma^{d_{0} \times(G / H)}}(t)=\left(\chi^{G} \zeta_{\sigma^{\left(d_{0}-1\right)} \times(G / H)}(t)\right)^{-1}=\left((1-t)^{-(-1)^{\left(d_{0}-1\right)}[G / H]}\right)^{-1} \\
=(1-t)^{-(-1)^{d_{0}[G / H]}}=(1-t)^{-\chi^{G}\left(\sigma^{\left.d_{0} \times(G / H)\right)}\right.} .
\end{gathered}
$$

The equation (14) has an interpretation similar to the one for "usual" (non-equivariant) higher order Euler characteristics. Set

$$
\mathbb{X}_{\left(X ; G_{O}, G_{B}\right)}^{(k)}(t):=\sum_{n \geq 0} \chi^{\left(k, G_{B}\right)}\left(X^{n} ; G_{O n}, G_{B}\right) \cdot t^{n}
$$


Let $G_{O}$ (regarded as a zero-dimensional finite space) be endowed with the natural action of the group $G_{O}$ and with the trivial action of the group $G_{B}$. Then one has $\chi^{\left(k, G_{B}\right)}\left(G_{O} ; G_{O}, G_{B}\right)=1$. Therefore

$$
\mathbb{X}_{\left(G_{O} ; G_{O}, G_{B}\right)}^{(k)}(t)=\left(\prod_{r_{1}, \ldots, r_{k} \geq 1}\left(1-t^{r_{1} r_{2} \cdots r_{k}}\right)^{r_{2} r_{3}^{2} \cdots r_{k}^{k-1}}\right)^{-1}
$$

and thus

$$
\mathbb{X}_{\left(X ; G_{O}, G_{B}\right)}^{(k)}(t)=\left(\mathbb{X}_{\left(G_{O} ; G_{O}, G_{B}\right)}^{(k)}(t)\right)^{\chi^{\left(k, G_{B}\right)}\left(X ; G_{O}, G_{B}\right)}
$$

\section{Equivariant generalized higher order Euler characteristics}

For a finite group $G$, let $K_{0}^{G}\left(\operatorname{Var}_{\mathbb{C}}\right)$ be the Grothendieck ring of complex quasi-projective $G$-varieties. By that we mean the free abelian group generated by the $G$-isomorphism classes $[X, G]$ (or $[X]$ for short) of complex quasi-projective varieties $X$ with $G$-actions modulo the relation: $[X, G]=$ $[Y, G]+[X \backslash Y, G]$ for a Zariski closed $G$-invariant subvariety $Y$ of $X$. The multiplication in $K_{0}^{G}\left(\operatorname{Var}_{\mathbb{C}}\right)$ is defined by the cartesian product with the diagonal $G$-action. Let $\mathbb{L} \in K_{0}^{G}\left(\operatorname{Var}_{\mathbb{C}}\right)$ be the class of the affine line $\mathbb{A}_{\mathbb{C}}^{1}$ with the trivial $G$-action.

Remark. Usually, in the definition of the Grothendieck ring of complex quasi-projective $G$-varieties, one adds one more relation: if $E \rightarrow X$ is a $G$ equivariant vector bundle of rank $n$, then $[E]=\left[\mathbb{A}_{\mathbb{C}}^{n} \times X\right]$. We do not need this relation for the construction. One can say that we use the Grothendieck ring denoted by $K_{0}^{\prime}, G\left(\operatorname{Var}_{\mathbb{C}}\right)$ in [5]. The same definition was used in [24]. An equation which holds in the equivariant Grothendieck ring $K_{0}^{G}\left(\operatorname{Var}_{\mathbb{C}}\right)$ defined here, holds in the "traditional" one as well.

There is a natural power structure over the (equivariant) Grothendieck $\operatorname{ring} K_{0}^{G}\left(\operatorname{Var}_{\mathbb{C}}\right)$. Its geometric definition is given in the same way as the usual power structure over the (non-equivariant) Grothendieck ring $K_{0}\left(\operatorname{Var}_{\mathbb{C}}\right)$ in [15]: for complex quasi-projective $G$-varieties $A_{i}, i=1,2, \ldots$, and $M$ one has

$$
\begin{aligned}
& \left(1+\left[A_{1}\right] t+\left[A_{2}\right] t^{2}+\ldots\right)^{[M]} \\
= & 1+\sum_{k=1}^{\infty} \sum_{\left\{k_{i}\right\}: \sum i k_{i}=k}\left[\left(\left(\prod_{i} M^{k_{i}}\right) \backslash \Delta\right) \times \prod_{i} A_{i}^{k_{i}} / \prod_{i} S_{k_{i}}\right] t^{k},
\end{aligned}
$$


where $\Delta$ is the "big diagonal" in $M^{\sum k_{i}}$, the symmetric groups $S_{k_{i}}$ act by the simultaneous permutations of the components of the corresponding factor $M^{k_{i}}$ in $M^{\sum k_{i}}=\prod_{i} M^{k_{i}}$ and on the components of $A_{i}^{k_{i}}$. One has to take into account that all summands in the right hand side of the equation (16) are $G$ invariant spaces. The proof of the necessary properties of the power structure is the same as in [15]. This power structure is induced by the $\lambda$-structure on $K_{0}^{G}\left(\operatorname{Var}_{\mathbb{C}}\right)$ defined by the Kapranov zeta-function. For a quasi-projective $G$-variety $X$, the series $(1-t)^{-[X]}$ is the equivariant Kapranov zeta-function of $X: \zeta_{[X]}(t):=1+[X] \cdot t+\left[S^{2} X\right] \cdot t^{2}+\left[S^{3} X\right] \cdot t^{3}+\ldots=(1-t)^{-[X]}$, where $S^{k} X=X^{k} / S_{k}$ is the $k$-th symmetric power of the $G$-variety $X$ with the natural $G$-action (see, e.g., 22], 24] for the non-equivariant case). The map $\chi^{G}: K_{0}^{G}\left(\operatorname{Var}_{\mathbb{C}}\right) \rightarrow A(G)$ is a $\lambda$-ring homomorphism.

In what follows we need the following statement.

Lemma 2 Let $p: E \rightarrow X$ be a G-equivariant vector bundle of rank $n$ such that for each $x \in X$ the action of the isotropy subgroup $G_{x}$ on the fibre $E_{x}=p^{-1}(x)$ is trivial. Then $[E]=\mathbb{L}^{n}[X]$.

Proof. Factorizing by the action of $G$ one gets the map $\check{p}: E / G \rightarrow X / G$ which is a vector bundle (due to the triviality of the action of $G_{x}$ on $E_{x}$ ). According to 25] the quotient $X / G$ can be covered by Zariski open subsets $U_{i}$ such that over each $U_{i}$ the fibre bunble $\check{p}$ is trivial. Therefore

$$
\check{p}^{-1}\left(U_{i}\right) \cong U_{i} \times \mathbb{A}_{\mathbb{C}}^{n}
$$

If $V_{i}=\pi^{-1}\left(U_{i}\right)$, where $\pi: X \rightarrow X / G$ is the canonical factorization map, then the trivialization (17) gives a trivialization of the bundle $p$ over $V_{i}$, i.e. an isomorphism between $p^{-1}\left(V_{i}\right)$ and $V_{i} \times \mathbb{A}_{\mathbb{C}}^{n}$ with the trivial $G$-action on $\mathbb{A}_{\mathbb{C}}^{n}$. This gives the statement.

In what follows we need the following properties of the power structure over the equivariant Grothendieck ring $K_{0}^{G}\left(\operatorname{Var}_{\mathbb{C}}\right)$.

Proposition 1 Let $A_{i}$ and $M$ be G-varieties, and let $A(t):=1+\left[A_{1}\right] t+$ $\left[A_{2}\right] t^{2}+\ldots \in K_{0}^{G}\left(\operatorname{Var}_{\mathbb{C}}\right)$. Then, for $s \geq 0$,

$$
\left(A\left(\mathbb{L}^{s} t\right)\right)^{[M]}=\left.(A(t))^{[M]}\right|_{t \mapsto \mathbb{L}^{s} t} .
$$


Proof. The coefficient at the monomial $t^{k}$ in the power series $(A(t))^{[M]}$ is a sum of the classes of the varieties of the form

$$
V=\left(\left(\prod_{i} M^{k_{i}}\right) \backslash \Delta\right) \times \prod_{i} A_{i}^{k_{i}} / \prod_{i} S_{k_{i}}
$$

with $\sum i k_{i}=k$. The corresponding summand $\widetilde{V}$ in the coefficient at the monomial $t^{k}$ in the power series $\left(A\left(\mathbb{L}^{s} t\right)\right)^{M}$ has the form

$$
\widetilde{V}=\left(\left(\prod_{i} M^{k_{i}}\right) \backslash \Delta\right) \times \prod_{i}\left(\mathbb{L}^{s i} A_{i}\right)^{k_{i}} / \prod_{i} S_{k_{i}} .
$$

There is the natural map $\widetilde{V} \rightarrow V$ which is a $G$-equivariant vector bundle of rank $s k$ satisfying the conditions of Lemma 2. By (2) one has $[\widetilde{V}]=\mathbb{L}^{s k}[V]$, what implies (18).

Proposition 1 together with the multiplicative properrty of the power structure $\left((A(t))^{[M][N]}=\left(A(t)^{[M]}\right)^{[N]}\right)$ implies the following statement.

Proposition 2 For a complex quasi-projective $G$-variety $X$ one has

$$
\zeta_{\mathbb{L}[X]}(t)=\zeta_{[X]}(\mathbb{L} t)
$$

Here we suggest an equivariant version of the generalized higher order Euler characteristic with values in the modification $K_{0}\left(\operatorname{Var}_{\mathbb{C}}\right)\left[\mathbb{L}^{s}\right]_{s \in \mathbb{Q}}$ of the equivariant Grothendieck ring of complex quasi-projective varieties.

Let $X$ be a smooth quasi-projective variety of dimension $d$ with commuting (algebraic) actions of two finite groups $G_{O}$ and $G_{B}$ (or equivalently with an action of the product $\left.G_{O} \times G_{B}\right)$.

Let us define the zero order equivariant generalized Euler characteristic of $\left(X ; G_{O}, G_{B}\right)$ by

$$
\left[X ; G_{O}, G_{B}\right]^{0, G_{B}}:=\left[X / G_{O}\right] \in K_{0}^{G_{B}}\left(\operatorname{Var}_{\mathbb{C}}\right) .
$$

For $g \in G_{O}$, let the set of the connected components of the fixed point set $X^{\langle g\rangle}$ consist of $N_{g}\left(C_{G_{O}}(g) \times G_{B}\right)$-orbits and let $X_{1}^{\langle g\rangle}, X_{2}^{\langle g\rangle}, \ldots, X_{N_{g}}^{\langle g\rangle}$ be the unions of the components of each of the orbits. For $1 \leq \alpha_{g} \leq N_{g}$ let $F_{\alpha_{g}}^{g}$ be the age $F_{x}^{g}$ at a point of $X_{\alpha_{g}}^{\langle g\rangle}$ (this age does not depend on the point $x \in X_{\alpha_{g}}^{\langle g\rangle}$. 
Definition: For a rational number $\varphi_{1} \in \mathbb{Q}$, the generalized orbifold Euler characteristic of weight $\varphi_{1}$ of $\left(X ; G_{O}, G_{B}\right)$ is defined by

$$
\begin{aligned}
& {\left[X ; G_{O}, G_{B}\right]_{\varphi_{1}}^{1, G_{B}}:=\sum_{[g] \in\left(G_{O}\right)_{*}} \sum_{\alpha_{g}=1}^{N_{g}}\left[X_{\alpha_{g}}^{\langle g\rangle} / C_{G_{O}}(g)\right] \cdot \mathbb{L}^{\varphi_{1} F_{\alpha_{g}}^{\langle g\rangle}}} \\
& =\sum_{[g] \in\left(G_{O}\right)_{*}} \sum_{\alpha_{g}=1}^{N_{g}}\left[X ; G_{O}, G_{B}\right]^{0, G_{B}} \cdot \mathbb{L}^{\varphi_{1} F_{\alpha_{g}}^{\langle g\rangle}} \in K_{0}^{G_{B}}\left(\operatorname{Var}_{\mathbb{C}}\right)\left[\mathbb{L}^{s}\right]_{s \in \mathbb{Q}} .
\end{aligned}
$$

For a subgroup $H_{O} \subset G_{O}$ and for an $H_{O} \times G_{B}$-invariant submanifold $Y \subset X$, let us define $\left[Y ; H_{O}, G_{B}\right]_{X, \varphi_{1}}^{1, G_{B}}$ by

$$
\left[Y ; H_{O}, G_{B}\right]_{X, \varphi_{1}}^{1, G_{B}}:=\sum_{[g] \in\left(H_{O}\right)_{*}} \sum_{\alpha_{g}=1}^{N_{g}}\left[Y_{\alpha_{g}}^{\langle g\rangle} / C_{H_{O}}(g)\right] \cdot \mathbb{L}^{\varphi_{1} F_{\alpha_{g}}^{\langle g\rangle}} \in K_{0}^{G_{B}}\left(\operatorname{Var}_{\mathbb{C}}\right)\left[\mathbb{L}^{s}\right]_{s \in \mathbb{Q}},
$$

where $Y_{\alpha_{g}}^{\langle g\rangle}=X_{\alpha_{g}}^{\langle g\rangle} \cap Y, F_{\alpha_{g}}^{\langle g\rangle}$ is the age of $g$ at a point of $X_{\alpha_{g}}^{\langle g\rangle}$.

Let $\underline{\varphi}=\left(\varphi_{1}, \varphi_{2}, \ldots\right)$ be a fixed sequence of rational numbers.

Definition: The equivariant generalized Euler characteristics of order $k$ of weight $\varphi$ of $\left(X ; G_{O}, G_{B}\right)$, as an element of $K_{0}^{G_{B}}\left(\operatorname{Var}_{\mathbb{C}}\right)\left[\mathbb{L}^{s}\right]_{s \in \mathbb{Q}}$, is defined recursively by

$$
\left[X ; G_{O}, G_{B}\right]_{\underline{\varphi}}^{k, G_{B}}:=\sum_{[g] \in\left(G_{O}\right)_{*}} \sum_{\alpha_{g}=1}^{N_{g}}\left[X_{\alpha_{g}}^{\langle g\rangle} ; C_{G}(g), G_{B}\right]_{X, \underline{\varphi}}^{(k-1), G_{B}} \cdot \mathbb{L}^{\varphi_{k} F_{\alpha g}^{\langle g\rangle}}
$$

where, for an $H_{0} \times G_{B}$-invariant submanifold $Y \subset X\left(H_{O} \subset G_{O}\right),\left[Y ; H_{O}, G_{B}\right]_{X, \underline{\varphi}}^{0, G_{B}}=$ $\left[Y ; H_{O}, G_{B}\right]^{0, G_{B}}$ is the zero order equivariant generalized Euler characteristic given by (201).

Remark. Iterating this definition one can write an equation for $\left[X ; G_{O}, G_{B}\right]_{\underline{\varphi}}^{k, G_{B}}$ analogues to (13). For a homomorphism $\phi: \mathbb{Z}^{k} \rightarrow G_{O}$, let the set of the connected components of the fixed point set $X^{\langle\phi\rangle}$ consist of $N_{\phi}\left(C_{G_{O}}(g) \times G_{B}\right)$ orbits and let $X_{1}^{\langle\phi\rangle}, X_{2}^{\langle\phi\rangle}, \ldots, X_{N_{\phi}}^{\langle\phi\rangle}$ be the unions of the components of each of the orbits. For $x \in X^{\langle\phi\rangle}$, let the shift $F_{x}^{\phi}$ be defined as $\sum_{i=1}^{k} \varphi_{i} F_{x}^{\phi\left(e_{i}\right)}$, where $e_{1}, \ldots, e_{k}$ is the standard basis of $\mathbb{Z}^{k}$. For $1 \leq \alpha_{\phi} \leq N_{g}$ let $F_{\alpha_{\phi}}^{\phi}$ be the 
shift $F_{x}^{\phi}$ at a point of $X_{\alpha_{\phi}}^{\langle\phi\rangle}$. One has:

$$
\left[X ; G_{O}, G_{B}\right]_{\underline{\varphi}}^{k, G_{B}}=\sum_{[\phi] \in \operatorname{Hom}\left(\mathbb{Z}^{k}, G_{O}\right) / G_{O}} \sum_{\alpha_{\phi}=1}^{N_{\phi}}\left[X^{\langle\phi\rangle} / C_{G_{O}}(\phi)\right] \cdot \mathbb{L}^{F_{\alpha_{\phi}}^{\phi}} .
$$

Theorem 2 Let $X$ be a (smooth) quasi-projective variety of dimension $d$ with commuting actions of two finite groups $G_{O}$ and $G_{B}$. Then

$\sum_{n \geq 0}\left[X^{n} ;\left(G_{O}\right)_{n}, G_{B}\right]_{\underline{\varphi}}^{k, G_{B}} \cdot t^{n}=\left(\prod_{r_{1}, \ldots, r_{k} \geq 1}\left(1-\mathbb{L}^{\Phi_{k}(\underline{r}) d / 2} \cdot t^{r_{1} r_{2} \cdots r_{k}}\right)^{r_{2} r_{3}^{2} \cdots r_{k}^{k-1}}\right)^{-\left[X ; G_{0}, G_{B}\right]_{\underline{\varphi}}^{k}}$

where

$$
\Phi_{k}\left(r_{1}, \ldots, r_{k}\right)=\varphi_{1}\left(r_{1}-1\right)+\varphi_{2} r_{1}\left(r_{2}-1\right)+\ldots+\varphi_{k} r_{1} r_{2} \cdots r_{k-1}\left(r_{k}-1\right) .
$$

Proof. The proof in [19] was by induction started from $k=1$, i.e. from the generalized orbifold case. The latter one was treated in [17]. One can easily see that the both proofs admit an action of an additional group $G_{B}$, i.e. all the subspaces are $G_{B}$-invariants. In particular, symmetric products participating in the proof of [17] carry the natural action of $G_{B}$. Using Propositions 1 and 2 the corresponding generating series can be written as exponents in terms of the power structure over the modification $K_{0}^{G_{B}}\left(\operatorname{Var}_{\mathbb{C}}\right)\left[\mathbb{L}^{s}\right]_{s \in \mathbb{Q}}$ of the equivariant Grothendieck ring of quasi-projective varieties.

\section{References}

[1] M. Atiyah, G. Segal. On equivariant Euler characteristics. J. Geom. Phys. 6 (1989), no.4, 671-677.

[2] V. Batyrev, D. Dais. Strong McKay correspondence, string-theoretic Hodge numbers and mirror symmetry. Topology 35 (1996), no.4, 901929.

[3] P. Berglund, T. Hübsch. A generalized construction of mirror manifolds. Nuclear Phys. B 393 (1993), 377-391. 
[4] P. Berglund, M. Henningson. Landau-Ginzburg orbifolds, mirror symmetry and the elliptic genus. Nuclear Phys. B 433 (1995), 311-332.

[5] F. Bittner. The universal Euler characteristic for varieties of characteristic zero. Compos. Math. 140 (2004), no.4, 1011-1032.

[6] L. Borisov. Class of the affine line is a zero divisor in the Grothendieck ring. Preprint arXiv:1412.6194.

[7] S. Bouc. Burnside rings. In: Handbook of algebra. Vol. 2. Edited by M. Hazewinkel. North-Holland, Amsterdam, 2000, 741-804.

[8] J. Bryan, J. Fulman. Orbifold Euler characteristics and the number of commuting $m$-tuples in the symmetric groups. Ann. Comb. 2 (1998), no.1, 1-6.

[9] J. Bryan, A. Morrison. Motivic classes of commuting varieties via power structures. J. Algebraic Geom. 24 (2015), no.1, 183-199.

[10] L. Dixon, J. Harvey, C. Vafa, E. Witten. Strings on orbifolds. I. Nuclear Phys. B 261 (1985), 678-686.

[11] L. Dixon, J. Harvey, C. Vafa, E. Witten. Strings on orbifolds. II. Nuclear Phys. B 274 (1986), 285-314.

[12] W. Ebeling, S.M. Gusein-Zade. Orbifold zeta functions for dual invertible polynomials. To appear in Proc. Edinb. Math. Soc. (2). ArXiv 1407.0154 .

[13] T. de Fernex, E. Lupercio, T. Nevins, B. Uribe. Stringy Chern classes of singular varieties. Adv. Math. 208 (2007), no.2, 597-621.

[14] E. Gorsky. Adams operations and power structures. Mosc. Math. J. 9 (2009), no.2, 305-323.

[15] S.M. Gusein-Zade, I. Luengo, A. Melle-Hernández. A power structure over the Grothendieck ring of varieties. Math. Res. Lett. 11 (2004), 4957.

[16] S.M. Gusein-Zade, I. Luengo, A. Melle-Hernández. Power structure over the Grothendieck ring of varieties and generating series of Hilbert schemes of points. Michigan Math. J. 54 (2006), no.2, 353-359. 
[17] S.M. Gusein-Zade, I. Luengo, A. Melle-Hernández. On the power structure over the Grothendieck ring of varieties and its applications. Proc. Steklov Inst. Math. 258 (2007), no.1, 53-64.

[18] S.M. Gusein-Zade, I. Luengo, A. Melle-Hernández. On an equivariant version of the zeta function of a transformation. Arnold Math. J. 1 (2015), no.2, 127-140.

[19] S.M. Gusein-Zade, I. Luengo, A. Melle-Hernández. Higher order generalized Euler characteristics and generating series. J. Geom. Phys. 95 (2015), 137-143.

[20] Y. Ito, M. Reid. The McKay correspondence for finite subgroups of SL $(3, \mathbb{C})$. In: Higher-dimensional complex varieties (Trento, 1994), 221240, de Gruyter, Berlin, 1996.

[21] F. Hirzebruch, Th. Höfer. On the Euler number of an orbifold. Math. Ann. 286 (1990), no.1-3, 255-260.

[22] M. Kapranov. The elliptic curve in the $S$-duality theory and Eisenstein series for Kac-Moody groups. ArXiv math.AG/0001005.

[23] I.G. Macdonald. The Poincaré polynomial of a symmetric product, Proc. Cambridge Philos. Soc. 58 (1962), 563-568.

[24] J. Mazur. Rationality of motivic zeta functions for curves with finite abelian group actions. J. Pure Appl. Algebra 217 (2013), no.7, 13351349 .

[25] J.P. Serre. Les espaces fibrés algébriques. In: Seminaire C. Chevalley, 2e année: 1958. Anneaux de Chow et applications, 1-37.

[26] R.P. Stanley. Enumerative Combinatorics. Vol.2. Cambridge Studies in Advanced Mathematics 62, 1999.

[27] T. tom Dieck. Transformation groups and representation theory. Lecture Notes in Mathematics, 766, Springer, Berlin, 1979.

[28] H. Tamanoi. Generalized orbifold Euler characteristic of symmetric products and equivariant Morava $K$-theory. Algebr. Geom. Topol. 1 (2001), 115-141. 
[29] W. Wang. Equivariant K-theory, wreath products, and Heisenberg algebra. Duke Math. J. 103 (2000), no.1, 1-23.

[30] W. Wang, J. Zhou. Orbifold Hodge numbers of wreath product orbifolds. J. Geom. Phys. 38 (2001), 152-169.

[31] E. Zaslow, Topological orbifold models and quantum cohomology rings. Commun. Math. Phys. 156 (1993), 301-331. 